\title{
Efficient Ship Maneuvering Prediction with Wind, Wave and Current Effects
}

\author{
Ying WANG ${ }^{\mathrm{a}}$, Wei DU ${ }^{\mathrm{b}}$, Guanghua $\mathrm{LI}^{\mathrm{c}}$, Zhuoyue $\mathrm{LI}^{\mathrm{a}, 1}$, Jingyao HOU${ }^{\mathrm{d}}$ and Haibao HU \\ a School of Marine Science and Technology, Northwestern Polytechnical University, \\ China \\ ${ }^{\mathrm{b}}$ Wuhan Second Ship Design and Research Institute, China \\ ${ }^{\mathrm{c}}$ Zhengzhou Institute of Electric and Mechanical Engineering, China \\ d China Ship Scientific Research Center, China
}

\begin{abstract}
Ship maneuvering simulation is an important tool to predict the kinetic and kinematic properties of a vessel. It can estimate the manoeuvrability of a vessel in advance to optimize the ship design and avoid possible problems. Environmental effects including wind, wave, current are very important disturbances from the nature, which can have great influence on the vessel. However, it is rather difficult to evaluate their influence currently. In this work, the wind, wave and current effects are implemented into the ship maneuvering model. Some typical results are demonstrated for verification. This tool is promising for engineering applications in ship design.
\end{abstract}

Keywords. Ship maneuvering, environmental effects, turning circle, hydrodynamic coefficient.

\section{Introduction}

With the increase of maritime transportation, the prediction of ships maneuverability especially under the environmental influences, such as wind, wave, current, etc., becomes a vital concern for maritime and coastal navigation control systems. Since the maneuverability ensures the ability of ship performance for safe navigation. The prediction of ship maneuvering becomes an important factor in ship engineering problems. It is important to evaluate the ship maneuverability in the design stage. Sarioz [1] presented the results of a real-time ship maneuvering simulation study examining the maneuvering performance of large tankers in the Bosphorus Strait. Vantorre [2] has provided a literature review on the ship-bank interaction with emphasis on experimental data and empirical formulas. Debaillon et al.[3] ont proposé un système de modélisation de squat d'un navire qui couple un modèle hydrodynamique et un modèle structurel. Sutulo[4]presented a frontier integral equation method applied for the calculation of inertia and damping characteristics of ship sections for multi-stage cases and inclined depths.

In this work, the wind, wave and current effects are implemented into the ship maneuvering model. Some typical results are demonstrated for verification. This tool is promising for engineering applications in ship design.

\footnotetext{
${ }^{1}$ Corresponding Author, Zhuoyue LI, School of Marine Science and Technology, Northwestern Polytechnical University, Xi'an 710072, China; Email: 2019200566@mail.nwpu.edu.cn.
} 


\section{Mathematical Formulations}

\subsection{Mathematical Formulations}

By applying the rigid body assumptions, the motion equations of ship can be written as [5-8]

$$
\boldsymbol{M}_{R B} \dot{\boldsymbol{v}}+\boldsymbol{C}_{R B} \boldsymbol{v}=\boldsymbol{\tau}_{R B}
$$

where $\boldsymbol{M}_{\mathrm{RB}}$ is the mass matrix. $\boldsymbol{v}$ is the velocity vector. $\boldsymbol{C}_{\mathrm{RB}}$ is the Coriolis and centripetal matrix. $\boldsymbol{\tau}_{R B}$ is the vector induced by external forces and moments. As seen in figure $1, \boldsymbol{V}$ and $V_{\text {env }}$ denote the direction of ship and environmental effect (wind, current, wave, etc.), $\psi$ the ship yaw angle and $\beta_{\text {env }}$ the angle of environmental forces in the global system. In the body-fixed coordinate,

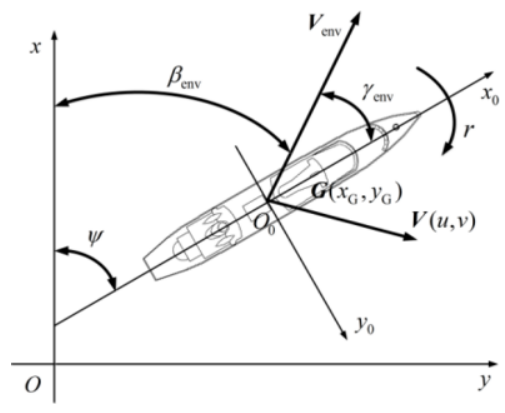

Figure 1. Coordinate frame.

The matrices of a 3DOF (degrees of freedom) model can be written as:

$$
\begin{aligned}
& \boldsymbol{M}_{R B}=\left[\begin{array}{ccc}
m & 0 & -m y_{G} \\
0 & m & m x_{G} \\
-m y_{G} & m x_{G} & I_{z}
\end{array}\right] \quad \boldsymbol{C}_{R B}=\left[\begin{array}{ccc}
0 & 0 & -m\left(x_{G} r+v\right) \\
0 & 0 & -m\left(y_{G} r-u\right) \\
m\left(x_{G} r+v\right) & m\left(y_{G} r-u\right) & 0
\end{array}\right] \\
& \boldsymbol{v}=\left[\begin{array}{lll}
u & v & r
\end{array}\right]^{T} \quad \boldsymbol{\tau}_{R B}=\left[\begin{array}{lll}
X & Y & N
\end{array}\right]^{T}
\end{aligned}
$$

where $m$ is the mass of ship. $x_{\mathrm{G}}$ and $y_{\mathrm{G}}$ are the position of the center of gravity. $I_{\mathrm{z}}$ is the moment of inertia around $z$ axis. $u, v$ and $r$ are the linear and angular velocities. $X, Y$, and $N$ are the forces and moment on the vessel. The dimensionless forms of $X, Y$, and $N$ are written as

$$
X^{\prime \prime}=\frac{X}{m g} \quad Y^{\prime \prime}=\frac{Y}{m g} \quad N^{\prime \prime}=\frac{N}{m g L}
$$

Therefore, the reduced maneuvering equations are

$$
\dot{u}-v r=g X^{\prime \prime} \quad \dot{v}+u r=g Y^{\prime \prime} \quad\left(L k_{z}^{\prime \prime}\right)^{2} \dot{r}+L x_{G}^{\prime \prime} u r=g L N^{\prime \prime}
$$

where $k_{z}^{\prime \prime}=\frac{1}{L} \sqrt{\frac{I_{z}}{m}}$ is the non-dimensional form of the radius of the turning. $x_{G}^{\prime \prime}=L^{-1} x_{G}$. The force and moment components on the ship $X^{\prime \prime}, Y^{\prime \prime}, N^{\prime \prime}$ include the hydrodynamic forces in calm water as well as various environmental ones due to wind, waves, currents, etc. There are two kinds of manner to express them, one is introduced by Prof. 
Abkowitz from MIT and the other one by the Mathematical Modeling Group (MMG) from Japan. The MMG model considers the ship system based on hull, rudder and propeller respectively. In this study, rather than the detailed interactions between the three parts, we focus on the whole ship. So here the Abkowitz model is a better choice. The hydrodynamic forces and moment can be written as [9-11]

$$
\begin{aligned}
X_{h y d}^{\prime \prime} & =X_{h y d}^{\prime \prime}(u, v, r, \dot{u}, \dot{v}, \dot{r}, T, \xi, c, \delta) \\
Y_{h y d}^{\prime \prime} & =Y_{h y d}^{\prime \prime}(u, v, r, \dot{u}, \dot{v}, \dot{r}, T, \xi, c, \delta) \\
N_{h y d}^{\prime \prime} & =N_{h y d}^{\prime \prime}(u, v, r, \dot{u}, \dot{v}, \dot{r}, T, \xi, c, \delta)
\end{aligned}
$$

where $T$ is the thrust of the propeller. $c$ is the flow velocity near the rudder. $\xi$ is the term caused by shallow water. $\delta$ is the rudder angle.

In this study, the Esso Bernicia 190,000dwt tanker is used, whose expanded hydrodynamic forces and moment can be shown as:

$$
\begin{aligned}
& g Y_{h y d}^{\| \prime}=Y_{\dot{v}}^{\| \prime} \dot{v}+\frac{1}{L} Y_{u v}^{\|} u v+\frac{1}{L} Y_{|v| v}^{\| \prime}|v| v+\frac{1}{L} Y_{|c| c \delta}^{\|}|c| c \delta+Y_{u r}^{\|} u r \\
& +\frac{1}{L} Y_{|c| c|\beta| \beta|\delta|}^{\prime \prime}|c| c|\beta| \beta|\delta|+Y_{T}^{\prime \prime} g T^{\prime \prime} \\
& +Y_{u r \xi}^{\prime \prime} u r \xi+\frac{1}{L} Y_{u v \xi}^{\prime \prime} u v \xi+Y_{\dot{v} \xi}^{\|} \dot{v} \xi+\frac{1}{L} Y_{|v| v \xi}^{\|}|v| v \xi \\
& +\frac{1}{L} Y_{|c| c|\beta| \beta|\delta| \delta \mid \xi}^{\prime \prime}|c| c|\beta| \beta|\delta| \xi \\
& g L N_{h y d}^{\prime \prime}=L^{2} N_{\dot{r}}^{\|} \dot{r}+N_{u v}^{\|} u v+L N_{|v| r}^{\prime \prime}|v| r+N_{|c| c \delta}^{\prime \prime}|c| c \delta+L N_{u r}^{\|} u r \\
& +N_{|c| c|c \beta \beta| \delta|| c \mid}^{\prime \prime}|c| c|\beta| \beta|\delta|+N_{T}^{\prime \prime} g T^{\prime \prime} \\
& +L N_{u r \xi}^{\prime \prime} u r \xi+L^{2} N_{\dot{r} \xi}^{\prime \prime} \dot{r} \xi+N_{u v \xi}^{\prime \prime} u v \xi+L N_{|v| r \xi}^{\prime \prime}|v| r \xi \\
& +N_{|c| c|\beta| \beta|\delta| \xi|\xi| c|\beta| \beta|\delta| \xi}^{\prime \prime}|c|
\end{aligned}
$$

where the first two lines are deep water effect and the last two lines the confinement effect. $X_{u}^{\prime \prime}, X_{u|u|}^{\prime \prime}, \ldots, Y_{\dot{v}}^{\prime \prime}, Y_{u v}^{\prime \prime}, \ldots, N_{\dot{r}}^{\prime \prime}, N_{u v}^{\prime \prime}, \ldots, N_{|c| c|\beta| \beta|| \xi}^{\prime \prime}$ are the hydrodynamic derivatives in [5], td the thrust deduction coefficient. $\beta=v / u . \xi=T_{d} /\left(h-T_{d}\right)$, where $h$ is the water depth. $T_{\mathrm{d}}$ is the draught of the ship.

\subsection{Environmental Effects}

According to Eq.l, environmental forces and moments can be incorporated into the term $\tau_{R B}$ as follows [12-16]:

$$
\tau_{R B}=\tau_{\text {hyd }}+\tau_{s}+\tau_{\text {wind }}+\tau_{\text {current }}+\tau_{\text {wave }}
$$

where $T_{\text {hyd }}$ is the hydrodynamic term, $t_{\mathrm{s}}$ the term of ship-to-ship interaction, $\tau_{\text {wind }}$, $\tau_{\text {current }}$ and $\tau_{\text {wave }}$ are the environmental terms, including wind, current and wave effect in this study.

\subsubsection{Wind Effect}

To correspond with the real situation, an experimental and statistical algorithm of the Oil Companies International Marine Forum (OCIMF) is utilized during the simulation. The OCIMF model is considered to be one of the better suitable methods for very large crude carriers (VLCC, vessels in the 150,000-500,000 dwt range). So, it is appropriate to be utilized for the Esso Bernicia 190,000dwt tanker of this work. 
The wind forces and moment of this model can be expressed as:

$$
X_{\text {wind }}=C_{X, \text { wind }} \frac{\rho_{a}}{7.6} V_{\text {wind }}^{2} A_{T} \quad Y_{\text {wind }}=C_{Y, \text { wind }} \frac{\rho_{a}}{7.6} V_{\text {wind }}^{2} A_{L} \quad N_{\text {wind }}=C_{N, \text { wind }} \frac{\rho_{a}}{7.6} V_{\text {wind }}^{2} A_{L} L
$$

where the curves of coefficients $C_{\mathrm{X} \text {,wind, }} C_{\mathrm{Y} \text {,wind }}, C_{\mathrm{N} \text {,wind }}$ are presented in [5]. $p_{\mathrm{a}}$ is the local air density, $A_{\mathrm{L}}$ and $A_{\mathrm{t}}$ the lateral and transverse projected area, $L$ the overall ship length. $A_{\mathrm{s}}$ in figure 1 , the angle of attack of the wind $\gamma_{\text {wind }}=\psi-\beta_{\text {wind }}-\pi$, where $\psi$ is the ship yaw angle. $\beta_{\text {wind }}$ is the angle of the wind in the global coordinate. The relative velocities can be written

$$
u_{r, \text { wind }}=u-V_{\text {wind }} \cos \left(\beta_{\text {wind }}-\psi\right) \quad v_{r, \text { wind }}=v-V_{\text {wind }} \sin \left(\beta_{\text {wind }}-\psi\right)
$$

\subsubsection{Current Effect}

Ocean currents can be formed by gravity, wind friction, heat exchange at sea surface, variation of water density, etc., both horizontally and vertically. For a 3DOF system, only the horizontal component is considered. In some areas, the current speed can reach $2-3 \mathrm{~m} / \mathrm{s}$ or more. The velocity field of ocean current is very close to homogeneous. Thereby uniform current is assumed for simplicity in this work. Like the wind effect (figure 1), the relative velocities of current can be expressed as:

$$
u_{r, \text { current }}=u-V_{\text {current }} \cos \left(\beta_{\text {current }}-\psi\right) \quad v_{r, \text { current }}=v-V_{\text {current }} \sin \left(\beta_{\text {current }}-\psi\right)
$$

\subsubsection{Wave Effect}

Waves on the ocean surface can be generated by many kinds of disturbances, such as wind, gravity, etc. They have a certain amount of randomness differing in height, duration and shape. Many approximations, including linear, 2nd-order and higher-order models, have been proposed by researchers [7]. When coming to the maneuvering problem, 2nd-order theory is usually sufficient to describe the response of most marine vehicles in the sea. The 'order' is expressed in the wave elevation $\xi(\mathrm{x}, \mathrm{t})$ by applying Stoke's expansion to second order.

$$
\zeta(x, t)=\sum_{i=1}^{N} A_{i} \cos \left(\omega_{e i} t-k_{i} x+\phi_{i}\right)+\sum_{i=1}^{N} \frac{1}{2} A_{i}^{2} \cos 2\left(\omega_{e i} t-k_{i} x+\phi_{i}\right)+O\left(A_{i}^{3}\right)
$$

Then the wave slopes for wave component $i$ can be calculated

$$
s_{i}(x, t)=\frac{d \xi_{i}(x, t)}{d x}=A_{i} k_{i} \sin \left(w_{e i} t-k_{i} x+\phi_{i}\right)+A_{i}^{2} k_{i}^{2} \sin 2\left(w_{e i} t-k_{i} x+\phi_{i}\right)+O\left(A_{i}^{3}\right)
$$

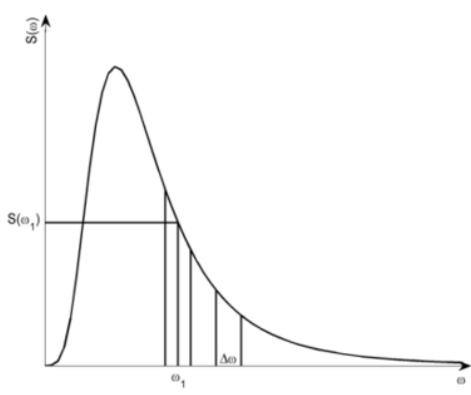

Figure 2. Wave spectrum with one peak. 
where the wave number $k_{\mathrm{i}}=w_{\mathrm{i}}^{2} / g . \Phi_{\mathrm{i}}$ is a random phase angle, which is uniformly distributed (figure 2). It is constant with time $[0,2 \pi]$. The encounter frequency $w_{e i}=w_{i}-U \cos \beta_{\text {wave }} w_{i}^{2} / g$ with $U$ the total speed of ship, $g$ the gravity acceleration and $\beta_{\text {wave }}$ the angle between ship heading and wave direction. $w_{i}$ is a random frequency of the spectral density function $S(s)$. As in figure 3, we only consider the wave spectra with one peak frequency. $S(w)$ is divided into $N$ intervals with length $A_{\text {s }}$. The wave amplitude $A_{i}=\sqrt{2 S\left(w_{i}\right) \Delta \omega}$. The wave spectra normally used are Neumann spectrum, Bretschneider spectrum, Pierson-Moskowitz (PM) spectrum, Torsethaugen spectrum, JONSWAP spectrum, etc. Among them, the JONSWAP spectrum is under the assumption of finite water depth and limited fetch, which make it suitable for the confinement problem. The modified PM spectrum is adopted here to consider a fully developed sea with large depth, no swell and unlimited fetch.

The wave forces and moment can be written

$$
\begin{gathered}
X_{\text {wave }}(x, t)=\sum_{i=1}^{N} \rho g B L T \cos \beta_{\text {wave }} s_{i}(t) \quad Y_{\text {wave }}(x, t)=-\sum_{i=1}^{N} \rho g B L T \sin \beta_{\text {wave }} s_{i}(t) \\
N_{\text {wave }}(x, t)=\sum_{i=1}^{N} \frac{1}{24} \rho g B L\left(L^{2}-B^{2}\right) \sin 2 \beta_{\text {wave }} s_{i}^{2}(t)
\end{gathered}
$$

Table 1. Hydrodynamic coefficients of the Esso Bernicia 190, 000dwt tanker

\begin{tabular}{|c|c|c|c|c|c|}
\hline No. & Coefficient & Value & No. & Coefficient & Value \\
\hline 1 & $X_{i}^{\prime \prime}$ & -0.0500 & 18 & $Y_{|v| v \xi}^{\prime \prime}$ & -1.5000 \\
\hline 2 & $X_{v r}^{\prime \prime}$ & 1.0200 & 19 & $N_{v r \xi}^{11}$ & -0.1200 \\
\hline 3 & $Y_{\dot{v}}^{\prime \prime}$ & -1.0200 & 20 & $Y_{|c| c \delta}^{\prime \prime}$ & 0.1761 \\
\hline 4 & $Y_{|c| c|\beta|}^{\prime \prime}$ & -2.1600 & 21 & $Y_{u v \xi}^{\prime \prime}$ & 0.0000 \\
\hline 5 & $Y_{T}^{\prime \prime}$ & 0.0400 & 22 & $N_{u v \xi}^{\prime \prime}$ & -0.2823 \\
\hline 6 & $N_{T}^{\prime \prime}$ & -0.0240 & 23 & $X_{c|c| \beta \delta}^{\prime \prime}$ & 0.1684 \\
\hline 7 & $N_{\dot{r}}^{\prime \prime}$ & -0.0728 & 24 & $N_{|c| c \delta}^{\prime \prime}$ & -0.0805 \\
\hline 8 & $Y_{|v| v}^{\prime \prime}$ & -2.4000 & 25 & $X_{|c| c \delta}^{\prime}$ & 0.0125 \\
\hline 9 & $N_{|v| r}^{\prime \prime}$ & -0.3000 & 26 & $N_{|c| c|c| \beta|\delta|}^{\prime \prime}$ & 0.6880 \\
\hline 10 & $X_{|v| v}^{\prime \prime}$ & 0.3000 & 27 & $Y_{|c| c|\beta| \beta|c| \xi}^{\|}$ & -0.1910 \\
\hline 11 & $Y_{u v}^{\prime \prime}$ & -1.2050 & 28 & $N_{|c| c|B||| \mid \xi \xi}^{\prime \prime}$ & 0.3440 \\
\hline 12 & $N_{u v}^{\prime \prime}$ & -0.4510 & 29 & $Y_{u r}^{\prime \prime}$ & 0.2480 \\
\hline 13 & $X_{\dot{u} \xi \xi}^{\prime \prime}$ & -0.0500 & 30 & $N_{u r}^{\prime \prime}$ & -0.2105 \\
\hline 14 & $Y_{\dot{v} \xi}^{\prime \prime}$ & -0.3780 & 31 & $X_{u|u|}^{\prime}$ & -0.0377 \\
\hline 15 & $Y_{u r \xi}^{\prime \prime}$ & 0.1598 & 32 & $N_{\dot{r} \xi}^{\prime \prime}$ & -0.0045 \\
\hline 16 & $N_{u r \xi \xi}^{\prime \prime}$ & -0.0416 & 33 & $X_{|u| u \xi}^{\prime \prime}$ & -0.0073 \\
\hline 17 & $X_{v r \xi}^{\prime \prime}$ & 0.3780 & 34 & $X_{c|c| \delta \delta}^{\prime \prime}$ & -0.1000 \\
\hline
\end{tabular}




\section{Results and Discussions}

The maneuvering equations are first tested to validate the feasibility and their accuracy. For the hydrodynamic coefficients, instead of the original ones, here we adopt the optimized ones from [5] to reduce the error of simulation.

\subsection{Turning Circle Test}

Normally the turning circle maneuver, zigzag maneuver, spiral maneuver, etc. are always conducted to evaluate the controllability and maneuverability of the vessel. In this study, the turning circle maneuver is first carried out for validation. The hydrodynamic coefficients can be found in table 1. Figure 3 is the comparison of calculated and experimental trajectories. The simulation results are close to that of experiments. The difference between the two is smaller than $10 \mathrm{~m}$. The surge velocity and yaw angle (figure 3) also agree with the experimental results.
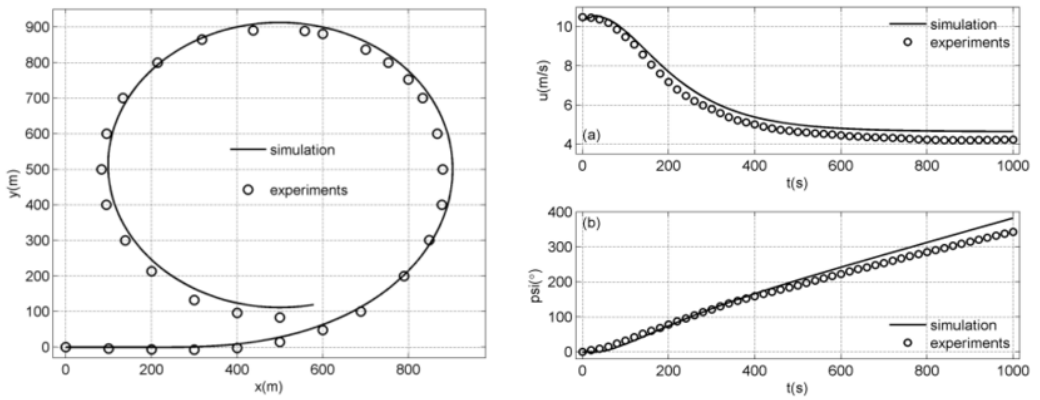

Figure 3. (a) Comparison of simulation and experimental trajectories in turning circle tests.

(b) Comparison of the surge velocity and yaw angle in turning circle maneuver.

\subsection{Turning Circle Tests With Environmental Effect}

The environmental terms above are validated using turning circle tests before applying to the ship overtaking simulation. As shown in figure 4 , wind, current and wave with $\beta$ $=0^{\circ}, 90^{\circ}, 180^{\circ}, 270^{\circ}$ are imposed during the simulation. The wind and current speeds are $60 \mathrm{knots}$ and $5 \mathrm{knots}$, respectively. The wave height and peak period of wave spectrum are $10 \mathrm{~m}$ and $5.0 \mathrm{~s}$. The trajectories indicate correct behaviors under the influences of wind, current and wave from all the directions. For the cases of wave effect, there exists a random phase angle 0 in the equations of wave elevation and wave slope. The results might change each time running the simulation. Overall, the maneuvering models can predict the ship trajectory and can be adopted in the formal applications. 

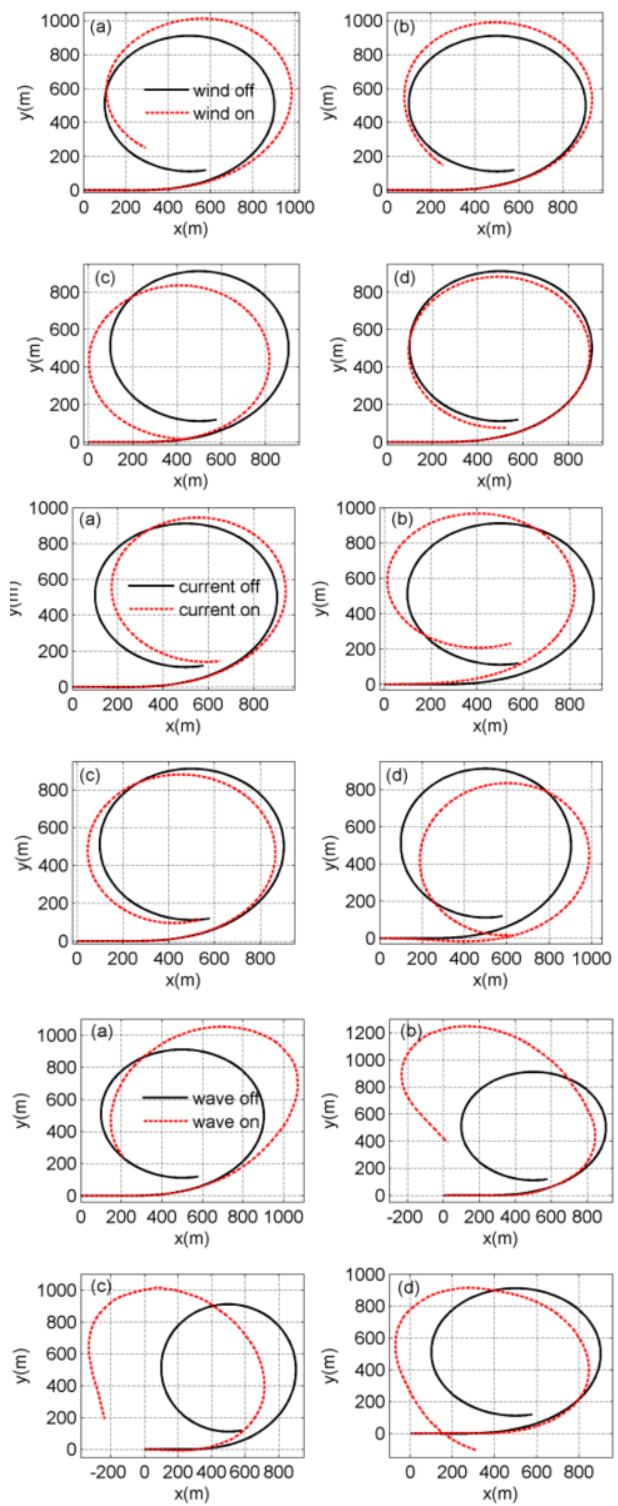

Figure 4. Comparison of turning circle tests with and without wind, current and wave effect. (a) $\beta=0$ \%; (b) $\beta=90^{\circ}$; (c) $\beta=180^{\circ}$; (d) $\beta=270^{\circ}$.

\section{Conclusions}

In this work the wind, wave and current effects are successfully implemented into the ship maneuvering model. The Esso Bernicia 190,000dwt tanker is adopted for the maneuvering simulations. The trajectories, surge velocity and yaw angle are validated with experimental results. Some typical results with wind, wave and current effects are 
demonstrated for verification. This tool is promising for engineering applications in ship design.

\section{Acknowledgments}

This work was funded by the Guangdong Basic and Applied Basic Research Foundation (2019A1515110863), Fundamental Research Funds for the Central Universities (3102020HHZY030004) and Shaanxi Provincial Key R\&D Program (2021KW-38).

\section{References}

[1] Sariöz K, Narli E 2003 Assessment of manoeuvring performance of large tankers in restricted waterways: a real-time simulation approach Ocean engineering 30(12): 1535-1551.

[2] Vantorre M, Delefortrie G, Eloot K, et al 2003 Experimental investigation of ship-bank interaction forces[C]. International Conference MARSIM.

[3] Debaillon P, Lataire E, Vantorre M 2009 Bank effect on ship squat[C]. International conference on Ship manoeuvring in shallow and confined water: bank effects. Royal Institution of Naval Architects 31-37.

[4] Sutulo S, Rodrigues J M, Soares C G 2010 Hydrodynamic characteristics of ship sections in shallow water with complex bottom geometry Ocean Engineering 37(10): 947-958.

[5] Du P, Ouahsine A, Tran K T, et al 2018 Simulation of the overtaking maneuver between two ships using the non-linear maneuvering model[J]. Journal of Hydrodynamics 30(5): 791-802.

[6] Du P, Ouahsine A, Sergent P, et al 2021 Investigation on Resistance, Squat and Ship-Generated Waves of Inland Convoy Passing Bridge Piers in a Confined Waterway[J]. Journal of Marine Science and Engineering 9(10): 1125 .

[7] Du P, Ouahsine A, Toan K T, et al 2017 Simulation of ship maneuvering in a confined waterway using a nonlinear model based on optimization techniques Ocean Engineering 142: 194-203.

[8] Du P, Ouahsine A, Sergent P, et al 2020 Resistance and wave characterizations of inland vessels in the fully-confined waterway[J]. Ocean Engineering 210: 107580.

[9] Du P, Wen J, Zhang Z, et al 2017 Maintenance of air layer and drag reduction on superhydrophobic surface Ocean Engineerin 130: 328-335.

[10] Du P, Ouahsine A, Sergent P 2018 Influences of the separation distance, ship speed and channel dimension on ship maneuverability in a confined waterway Comptes rendus mécanique 346(5): 390401.

[11] Du P, Ouahsine A, Hoarau Y. 2018 Solid body motion prediction using a unit quaternion-based solver with actuator disk Comptes Rendus Mecanique 346(12): 1136-1152.

[12] Haibao H, Peng D, Feng Z, et al 2015 Effect of hydrophobicity on turbulent boundary layer under water Experimental Thermal and Fluid Science 60: 148-156.

[13] Song D, Song B, Hu H, et al 2018 Effect of a surface tension gradient on the slip flow along a superhydrophobic air-water interface Physical Review Fluids 3(3): 033303.

[14] Ding D, Ouahsine A, Xiao W, et al 2021 CFD/DEM coupled approach for the stability of caisson-type breakwater subjected to violent wave impact Ocean Engineering 223: 108651.

[15] Xie, Z., Zhang, Y., Zhou, J., Zhu, W 2021 Theoretical and experimental research on the micro interface lubrication regime of water lubricated bearing. Mech. Syst. Signal Process 151, 107422.

[16] Xie, Z., Zhu, W 2022 An investigation on the lubrication characteristics of floating ring bearing with consideration of multi-coupling factors. Mech. Syst. Signal Process 162, 108086. 